\title{
Nanoparticles show potential to retard bradyzoites in vitro formation of Toxoplasma gondii
}

\author{
Oluyomi Stephen Adeyemi ${ }^{1,2}$, Yuho Murata ${ }^{1}$, Tatsuki Sugi ${ }^{1}$, Yongmei Han ${ }^{1}$ and Kentaro Kato \\ ${ }^{1}$ National Research Center for Protozoan Diseases, Obihiro University of Agriculture and Veterinary Medicine, Inada-cho, Obihiro, Japan; \\ ${ }^{2}$ Medicinal Biochemistry and Toxicology Laboratory, Department of Biochemistry, Landmark University, Ipetu Road, Nigeria
}

\begin{abstract}
Toxoplasmosis is a common parasitic disease caused by Toxoplasma gondii (Nicolle et Manceaux, 1908), an obligate parasite capable of infecting a range of cell types in almost all warm-blooded animals. Upon infecting an intermediate host, the parasites differentiate into tachyzoites which rapidly infect host tissues. Usually, the invading parasites are cleared by the immune system and administered drugs, but some tachyzoites differentiate into bradyzoites forming tissue cysts. These tissue cysts could serve as a source for re-infection and exacerbations. Currently, treatment for toxoplasmosis is limited and, moreover, there are no drugs for treating the cystic stage thus rendering toxoplasmosis a global burden. Recently, we demonstrated that inorganic nanoparticles showed promising activity against the tachyzoite stage $T$. gondii. In the present study, we evaluated nanoparticles for effect on bradyzoite formation in vitro. Data revealed that the nanoparticles limited bradyzoite burden in vitro. Further, the nanoparticles decreased the bradyzoite-specific BAG-1 promoter activity relative to the untreated control under a bradyzoite-inducing culture condition, even though this reduction in BAG-1 promoter activity waned with increasing concentrations of nanoparticles. In contrast, a parallel experiment under normal cell culture conditions showed that the nanoparticle treatment mildly increased the BAG-1 promoter activity relative to the untreated control. Taken together, the findings are evidence that nanoparticles not only possess anti-tachyzoite potential but they also have anti-bradyzoite potential in vitro.
\end{abstract}

Keywords: cyst, infection, medicinal biochemistry, nanomedicine, toxoplasmosis

Toxoplasma gondii (Nicolle et Manceaux, 1908) is the causative agent of toxoplasmosis, which affects nearly one-third of the human population (Black and Boothroyd 2000). In healthy individuals, the primary infection can be asymptomatic, but may prove fatal in those who are pregnant or immunocompromised (Black and Boothroyd 2000, Skariah et al. 2010). The parasite transits between the tachyzoite and bradyzoite (cystic) forms, thus making the life cycle complex (Black and Boothroyd 2000). Current treatments for toxoplasmosis include the use of pyrimethamine, sulfadiazine and clindamycin (during pregnancy spiramycin), which can suppress the active infection (Kamau et al. 2012); however, therapy that targets the dormant cyst stages is lacking. The bradyzoite-containing cysts of $T$. gondii are responsible for chronic toxoplasmosis. The cysts are sequestered in tissues and upon reactivation, can be life-threatening particularly for the immunocompromised patients. Many potential new molecular targets have been identified (Maubon et al. 2010), but, no drug candidate with anti-cystic activity has been reported to date. Therefore, the discovery of new therapeutic agents that are effective on cysts is an important challenge in toxoplasmosis control.
Accordingly, new approaches in the treatment of toxoplasmosis are needed. Recently, the application of nanotechnological approaches for biomedical purposes has rapidly expanded (Curtis and Wilkonson 2001) and nanoparticles are becoming attractive as potential treatment options for various diseases (Debbage 2009, Allahverdiyev et al. 2011). The small size and large ratio of surface area to volume are unique characteristics of nanoparticles, which can be exploited for medicinal value. These properties enhance reactivity and aids easy cellular penetration which might result in interaction with cellular biomolecules in a number of ways (Adeyemi et al. 2017). We recently showed that inorganic nanoparticles have strong activity against the tachyzoites form of $T$. gondii in vitro and these nanoparticles significantly suppressed parasite growth by $\geq 80 \%$ possibly through generation of reactive oxygen species (Adeyemi et al. 2017). Herein, we sought to determine whether nanoparticles can prevent and/or eliminate the cystic forms of $T$. gondii in vitro.

\section{MATERIALS AND METHODS}

Inorganic nanoparticles (NPs) including gold (AuNP, $5 \mathrm{~nm}$ ), silver (AgNP, $10 \mathrm{~nm})$ and platinum (PtNP, $3 \mathrm{~nm})$ were supplied

Address for correspondence: K. Kato, National Research Center for Protozoan Diseases, Obihiro University of Agriculture and Veterinary Medicine, Inada-cho, Obihiro, Hokkaido 080-8555, Japan. Phone: +81 15549 5645; Fax: +81 15549 5646; E-mail: kkato@obihiro.ac.jp 

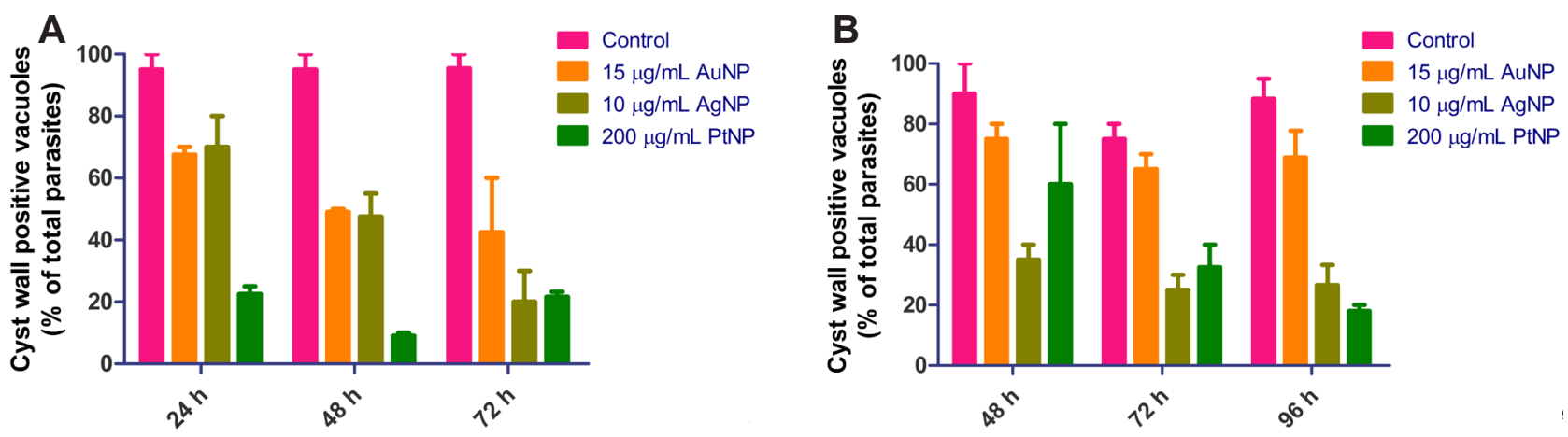

Figs. 1. Frequency of bradyzoites per treatment. A - effect of inorganic nanoparticles (NPs) on cysts in vitro. ME49 strain of Toxoplasma gondii (Nicolle et Manceaux, 1908) was inoculated onto confluent HFF monolayers and cyst formation was allowed to proceed under bradyzoite-inducing culture conditions. After $72 \mathrm{~h}$ ( $75-88 \%$ of total parasite was cyst wall positive), NPs were added and the incubation continued for 24,48 , and $72 \mathrm{~h}$ under bradyzoite-inducing culture conditions; $\mathbf{B}$ - effect of NPs on cyst wall formation. ME49 strain of $T$. gondii was inoculated onto confluent HFF monolayers. NPs were added $24 \mathrm{~h}$ after parasite inoculation and the incubation continued for 48, 72, and $96 \mathrm{~h}$ under bradyzoite-inducing culture conditions. For explanation - see text, page 2.

The graph shows averages for independent experiments performed in triplicates and normalised to the number of cysts present in the untreated control sample. Data are presented as the mean $\pm \mathrm{SEM}$; statistical significance relative to the control is indicated by a $p$ value of $<0.05$.

by Sigma-Aldrich (St. Louis, MO, USA). The nanoparticles were used as supplied after they were evaluated to confirm the supplier's specifications. All reagents were of analytical grade and used as supplied unless otherwise stated.

\section{In vitro effect of NPs on cyst formation as assessed by use of an} immunofluorescence assay

ME49 strain of Toxoplasma gondii strain was used for this study unless otherwise indicated. The parasite was maintained by repeated passages in monolayers of human foreskin fibroblast (HFF) cells cultured in Dulbecco's Modified Eagle Medium (DMEM; Nissui, Tokyo, Japan) and supplemented with GlutaMAX ${ }^{\mathrm{TM}}$-I (Gibco, Thermo Fisher Scientific, Waltham, MA, USA), $10 \%(\mathrm{v} / \mathrm{v})$ fetal calf serum (FCS; Gibco, Thermo Fisher Scientific, Waltham, MA, USA) and penicillin and streptomycin (100 U.mL ${ }^{-1}$; Biowhittaker, Leicestershire, UK).

In order to obtain parasite suspension, host cells infected with $T$. gondii tachyzoites were lysed by passing them through a 27-gauge needle. The cell lysates were then filtred and washed three times in fresh culture medium to yield a tachyzoite suspension free of host cell debris. The parasite density was measured with a hemocytometre and adjusted for in vitro experimental infection analysis.

To assess the effect of the NPs on cyst formation, we examined in vitro differentiation by using an immunofluorescence assay (IFA) under two different conditions. For bradyzoite induction, ME49-infected HFF cells were treated with $1 \%$ FCS in DMEM containing $25 \mathrm{mM}$ HEPES [pH 8.1] but no $\mathrm{NaHCO}_{3}$ from $24 \mathrm{~h}$ after parasite inoculation, in humid air, for $72 \mathrm{~h}$. From our preliminary experiments, cyst formation in vitro was achieved (>50\% cyst wall positive of total parasites) after at least a 48-hr incubation under these bradyzoite-inducing cell culture conditions. NP concentrations were selected on the basis of a previously published report of the effective concentrations for tachyzoite death (Adeyemi et al. 2017).

First, we sought to determine the effect of NPs on cysts in vitro. Briefly, ME49 strain of T. gondii was inoculated onto confluent HFF monolayers on coverslips at a multiplicity of infection
(MOI) of 0.5. The coverslips were incubated at $37^{\circ} \mathrm{C}$ and $5 \% \mathrm{CO}_{2}$ for $24 \mathrm{~h}$ to allow for invasion and infection. The infection medium was then removed and replaced with bradyzoite-inducing medium ( $\mathrm{pH} 8.1$, containing 1\% FCS in DMEM). Further incubation at $37^{\circ} \mathrm{C}$ and $0 \% \mathrm{CO}_{2}$ was then carried out for $72 \mathrm{~h}$. Thereafter, the NPs were added in fresh bradyzoite-inducing medium and the coverslips were incubated at $37^{\circ} \mathrm{C}$ and $0 \% \mathrm{CO}_{2}$ for 24,48 and $72 \mathrm{~h}$. At each of these time points, the coverslips were fixed with $4 \%$ paraformaldehyde (PFA) and stained with an anti-CST-1 Salmon E monoclonal antibody as described elsewhere (Tomita et al. 2013). The total parasites were detected by staining with rabbit anti- $T$. gondii $\mathrm{RH}$ strain polyclonal antibodies $(1: 1,000)$ (Bio-Rad Laboratories, Hercules, CA, USA) and nuclear staining was achieved by using DAPI (4',6-diamidino-2-phenylindole, 1 : 1,000). Secondary staining was achieved by antibody solutions containing 1 : 1,000 ALEXA 488 goat anti-mouse (green signal for cyst wall) and ALEXA 594 goat anti-rabbit (red signal for total parasites) in $2 \%$ FCS-PBS. At least 25 random views were counted using a Nikon Eclipse E400 (Tokyo, Japan). The number of bradyzoites was estimated as a percentage of the total parasites.

In the second experimental condition, we assessed whether the NPs could prevent cyst wall formation in vitro. Briefly, ME49 strain of $T$. gondii was inoculated onto confluent HFF monolayers on coverslips at a MOI of 0.5 . The coverslips were incubated at $37^{\circ} \mathrm{C}$ and $5 \% \mathrm{CO}_{2}$ for $24 \mathrm{~h}$ The NPs were added in bradyzoite-inducing medium $(\mathrm{pH} 8.1) 24 \mathrm{~h}$ after parasite inoculation and the coverslips were further incubated at $37^{\circ} \mathrm{C}$ and $0 \% \mathrm{CO}_{2}$ for 48,72 and $96 \mathrm{~h}$. At each of these time points, the coverslips were fixed with 4\% PFA and stained with an anti-CST-1 Salmon E monoclonal antibody as described above.

\section{In vitro effect of NPs on bradyzoite-specific BAG-1 promoter activity}

In this experiment, we utilised a parasite expressing luciferase activity, PLK/DLUC_1C9 (Sugi et al. 2014), in a microplate screening assay format. Briefly, HFF cells were seeded in a solid white 96-well culture plate (Nunc; Fisher Scientific, Pittsburgh, USA) for $72 \mathrm{~h}$. The HFF cells were then infected with 10,000 
A

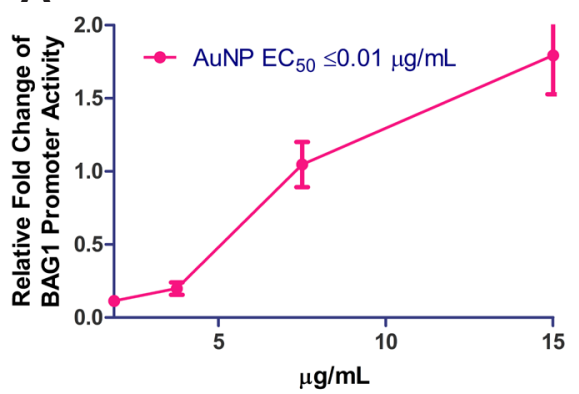

D

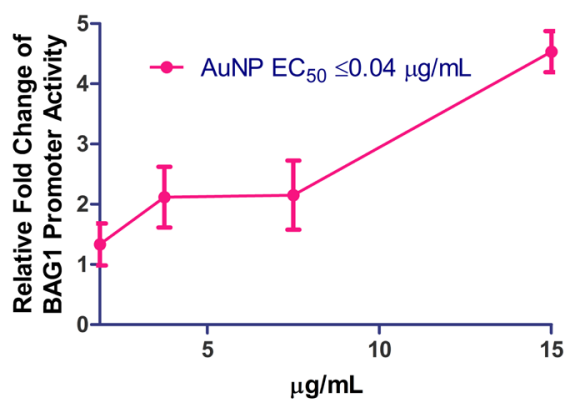

B

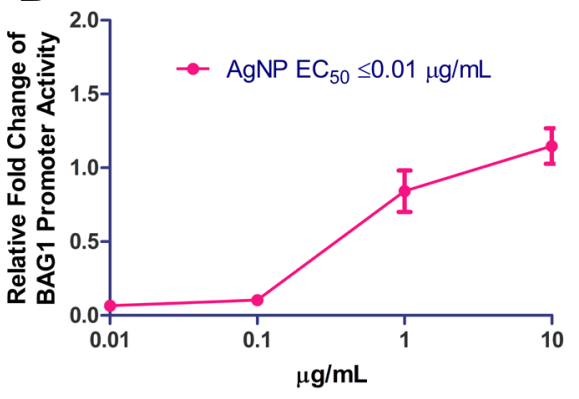

$E$

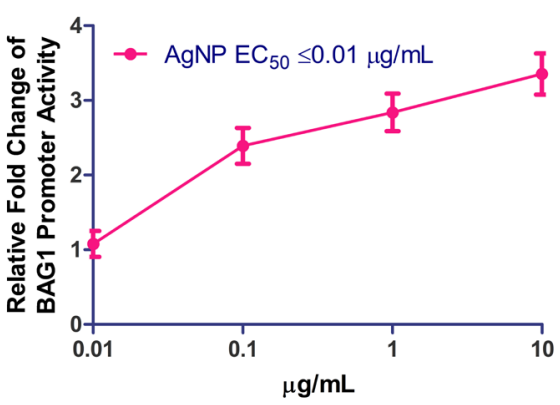

C

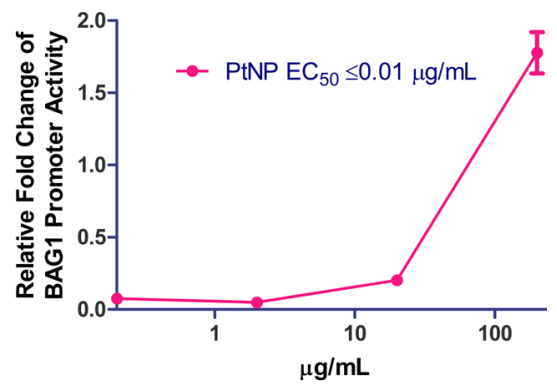

$\mathbf{F}$

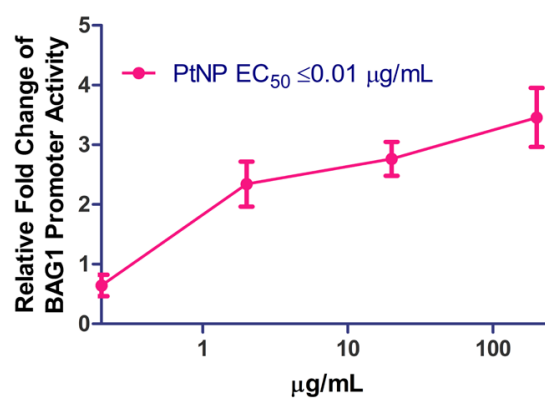

Fig. 2. A-C -48 hpi, bradyzoite-inducing culture conditions. In vitro effect of NPs on bradyzoite-specific BAG-1 promoter activity. HFF cells were infected with 10,000 parasites (PLK/DLUC_1C9) per well. Treatment with NPs (concentration range, $0.01-200 \mu \mathrm{g} /$ $\mathrm{mL}$ ) was initiated at $24 \mathrm{~h}$ post-inoculation and continued for $48 \mathrm{~h}$ under bradyzoite-inducing culture conditions; D-F - 48 hpi, normal cell culture conditions. To normalise for bradyzoite formation, the parasite expressed Renilla luciferase under the control of the $\alpha$-tubulin promoter. Data are presented as the mean \pm SEM. The experiment was performed three times independently. $\alpha$ is significant at $p$ $<0.05$ relative to the control.

parasites (PLK/DLUC_1C9) per well. Treatment with NPs (concentration range, $0.01-200 \mu \mathrm{g} / \mathrm{mL}$ ) was initiated at $24 \mathrm{~h}$ post-inoculation and continued for $48 \mathrm{~h}$ under bradyzoite-inducing culture conditions or normal cell culture conditions. Luciferase activity was measured by using the Dual-Glo Luciferase Assay System (Promega, Madison, WI, USA). PLK/DLUC_1C9 expressed firefly luciferase under the control of the bradyzoite-specific BAG-1 promoter. To normalise for bradyzoite formation, the parasite expressed the Renilla luciferase under the control of the $\alpha$-tubulin promoter. A known bradyzoite-inducing agent, ammonium pyrrlidinedithiocarbamate (PDTC; Sigma-Aldrich, St. Louis, MO, USA) served as a positive control.

\section{Data analysis}

Data were analysed using one-way ANOVA (GraphPad Software Inc., San Diego, CA, USA) and are presented as the mean \pm SEM. Differences among the group means were determined by using the Tukey post-hoc test; $p$ values $<0.05$ were considered to be significant. The concentration of the nanoparticles causing a $50 \%$ reduction in BAG-1 promoter activity (i.e. $\mathrm{EC}_{50}$ value) was estimated by using Prism 5 (GraphPad; CA, USA); non-linear regression analysis was used to fit the curve.

\section{RESULTS}

NPs retard tachyzoite to bradyzoite conversion in vitro

Under the first experimental condition, AuNP and AgNP treatment led to a time-dependent reduction in cyst wall positive vacuoles (Fig. 1A). For PtNP, the reduction in cyst wall positive vacuoles do not seem to be time-dependent.
Altogether, the finding indicate the potential of NPs to reduce bradyzoite burden.

Under the second experimental condition, we found that the NPs significantly reduced parasite cyst wall formation in vitro (Fig. 1B). Bradyzoite formation was retarded but not in a time-dependent manner. This further confirms the anti-bradyzoite potential of the NPs.

\section{NPs decrease BAG-1 promoter activity}

Using a parasite expressing luciferase activity, PLK/ DLUC_1C9, we tested the effect of NPs on BAG-1 promoter activity under two different conditions. Under the first condition, whereby the parasite was cultured under bradyzoite-inducing culture conditions, treatment with NPs decreased BAG-1 promoter activity relative to the untreated control (Fig. 2A-C). However, this reduction in BAG-1 promoter activity waned with increasing concentrations of NPs. In a parallel experiment under normal cell culture conditions, NP treatment increased the BAG-1 promoter activity only mildly relative to the untreated control (Fig. 2D-F). Under the same conditions, $80 \mu \mathrm{M}$ PDTC increased the BAG-1 promoter activity by $>3$-fold relative to the control (data not shown), thus validating the assay. Furthermore, the treatment with NPs significantly reduced the total parasite number (Fig. 3A-F), suggesting that the anti-bradyzoite effect of the NPs is linked to its anti-tachyzoite potential. 
A

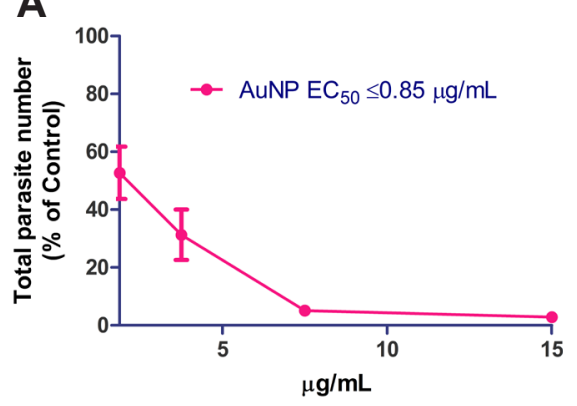

D

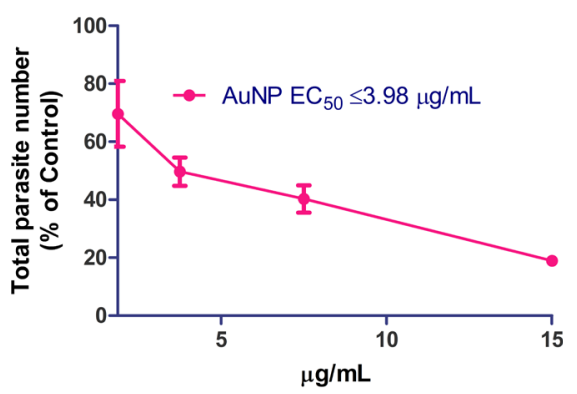

B

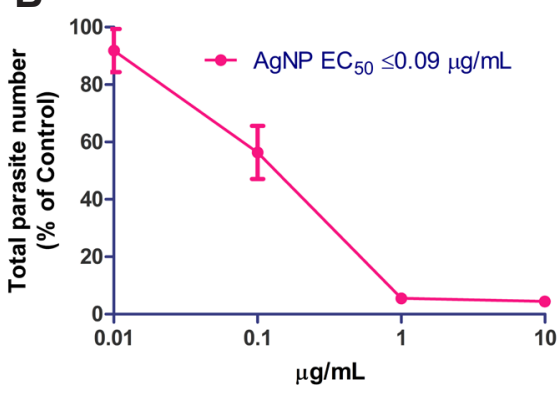

E

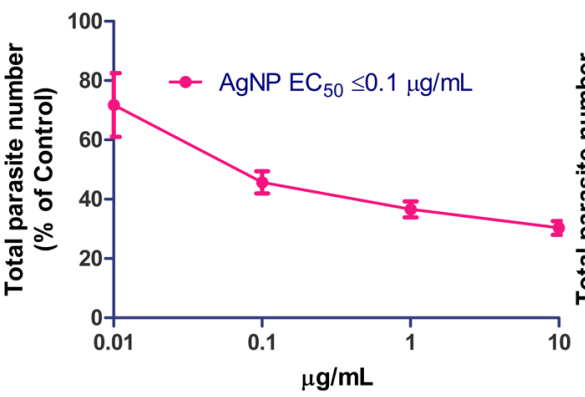

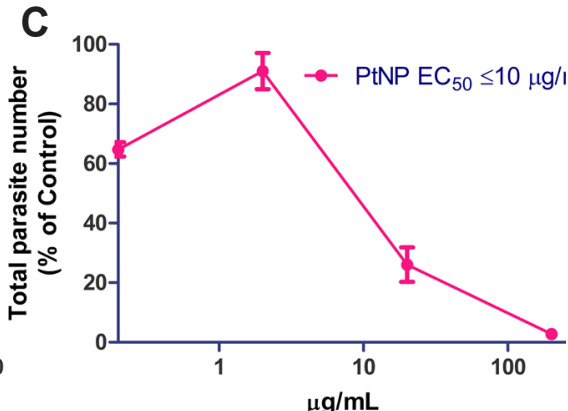

$\mathbf{F}$

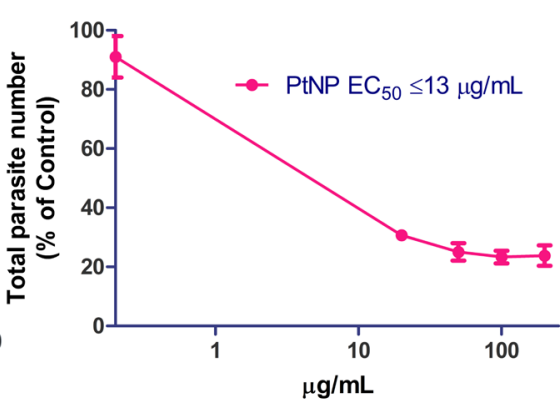

Fig. 3. A-C -48 hpi, bradyzoite-inducing culture conditions. In vitro effect of NPs on total parasite number HFF cells were infected with 10,000 parasites (PLK/DLUC_1C9) per well. Treatment with NPs (concentration range, 0.01-200 $\mu \mathrm{g} / \mathrm{mL}$ ) was initiated at $24 \mathrm{~h}$ post-inoculation and continued for $48 \mathrm{~h}$ under bradyzoite-inducing culture conditions; D-F -48 hpi normal cell culture conditions. Total parasite number is the same as the luminescence of Renilla luciferase. Data are presented as the mean \pm SEM. PLK/DLUC_1C9 expressed firefly luciferase under the control of the bradyzoite-specific BAG-1 promoter. To normalise for bradyzoite formation, the parasite expressed the Renilla luciferase under the control of the $\alpha$-tubulin promoter. A known bradyzoite-inducing agent, ammonium pyrrlidinedithiocarbamate (PDTC; Sigma-Aldrich) served as a positive control. The experiment was performed three times independently.

\section{DISCUSSION}

The large global burden of toxoplasmosis and the lack of effective therapeutic options underscore the urgent need for better anti-T. gondii drugs. Current therapy for toxoplasmosis suppresses the active infection but does not cure the latent infection (Black and Boothroyed 2000, Boothroyd and Dubremetz 2008). An ideal anti-T. gondii drug would be potent, nontoxic and eliminate the latent infection (Kamau et al. 2012). Recently, we demonstrated that inorganic nanoparticles showed promising activity against the tachyzoite stage of Toxoplasma gondii (Adeyemi et al. 2017). In the present study, we provide evidence that NPs have anti-bradyzoite potential.

First, we evaluated the effect of NPs on already formed cysts in vitro. In this experiment, tachyzoites-infected cells were cultured under bradyzoite-inducing culture conditions for $72 \mathrm{~h}$ before treatment with the NPs. The NPs caused a dramatic and significant reduction in the percentage of bradyzoites relative to the control. We also tested whether the NPs could prevent cyst formation and found that NPs have the capacity to limit bradyzoite formation, even under bradyzoite-inducing culture conditions. Taken together, these findings suggest that NPs have anti-bradyzoite capability.

We utilised a dual luciferase reporting parasite strain (PLK/DLUC_1C9) (Sugi et al. 2014), which is capable of stage interconversion in vitro and expresses firefly luciferase under the control of the bradyzoite-specific BAG-1 promoter. Our findings showed that, under bradyzoite-in- ducing culture conditions, treatment with NPs reduced BAG-1 promoter activity compared with the control, suggesting that NPs can reduce bradyzoite formation. Although the reduction in BAG-1 promoter activity waned as the NP concentration was increased, the impact of NPs on BAG-1 promoter activity suggests that NPs can retard bradyzoite formation.

In a parallel experiment with the PLK/DLUC_1C9 parasite, we found that under normal cell culture conditions, NP treatment mildly elevated BAG-1 promoter activity relative to the untreated control. Under the same conditions, PDTC, a known bradyzoite-inducing agent (Sugi et al. 2014), caused a significant (>3-fold) increase in BAG-1 promoter activity (data not shown). The increase in BAG-1 promoter activity could be a residual effect, which may be due to the stress imposed by treatment with NPs. However, the BAG-1 promoter activity increased with increasing concentrations of the NPs. We previously showed that oxidative stress contributes to the $T$. gondii killing capacity of NPs (Adeyemi et al. 2017). Therefore, it is plausible that the NPs acted in an off-target manner to stimulate BAG-1 promoter activity.

Previous studies have demonstrated that exogenous stress, including inhibition of replicating tachyzoites, could stimulate cyst wall formation in T. gondii (see Bohne et al. 1994, Weiss et al. 1998, Skariah et al. 2010). This fact further strengthens our reasoning that NP-induced restriction of replicating tachyzoites under both normal and 
bradyzoite-inducing culture conditions might have triggered the mild bradyzoite formation.

Furthermore, the role of stress induced by NP treatment is underscored if we consider that, under bradyzoite-inducing culture conditions, the reduction in BAG-1 promoter activity gradually waned with increasing NP concentrations. This may indicate that increasing the concentration of the NPs effectively reduced the total parasite number, but failed to concomitantly decrease the BAG-1 promoter activity due to the stress imposed by the NP treatment. Beyond this, data may also indicate that NPs have activity against both the tachyzoite and bradyzoite forms of $T$. gondii.

Taken together, our data provide evidence for the anti-bradyzoite potential of NPs but demonstrate that the anti-T. gondii potential of NPs may not necessarily preclude bradyzoite formation. Our findings support the anti-parasitic action of NPs and are consistent with other reports that have demonstrated the anti-protozoal (Ahmad et al. 2015, Rahul et al. 2015, Saad et al. 2015, Yah and Simate 2015, Saini et al. 2016), including anti-T. gondii (Leyke et al. 2012, Adeyemi et al. 2017, 2018a), potential of NPs.

However, to our knowledge, this is the first study to provide evidence in support of the anti-bradyzoite potential of NPs. Moreover, the effective doses ( $\mathrm{EC}_{50}$ values) of nanoparticles reported in the present study are far below the dose $(\geq 40 \mu \mathrm{g} / \mathrm{mL})$ required to cause cytotoxicity in HFF monolayers (Adeyemi et al. 2017).

In the separate investigations, in vitro cellular toxicity by gold, silver or platinum nanoparticles have been reported at doses $\geq 100 \mu \mathrm{g} / \mathrm{mL}$ (Jiao et al. 2014, Adeyemi and Sulaiman 2015, Adeyemi et al. 2018a,b). This dose is higher than the effective doses of nanoparticles used in the present study. Additionally, in vivo toxicity studies have shown that gold, silver and platinum had oral $\mathrm{LD}_{50}$ of $\geq 2000 \mathrm{mg} /$ $\mathrm{kg}, \geq 1,000 \mathrm{mg} / \mathrm{kg}$ and $\geq 1,000 \mathrm{mg} / \mathrm{kg}$ body weight, respectively (Pokharkar et al. 2009, Maneewattanapinyo et al. 2011, Adeyemi and Faniyan 2014, Adeyemi et al. 2014, 2016, Sulaiman et al. 2015).

In conclusion, although the NPs did not completely prevent bradyzoite formation, our data show that NPs have the capacity to limit the bradyzoite burden in vitro. These novel findings not only support the anti-parasitic potential of NPs but further strengthen the prospects of exploring NPs as promising alternative anti-parasitic agents.

Acknowledgements. The research was funded through a JSPS Fellowship to O.S. Adeyemi. This study was also supported by grants-in-aid for Young Scientists, Exploratory Research and Scientific Research on Innovative Areas (3407) from the Ministry of Education, Culture, Science, Sports and Technology (MEXT) of Japan, by the Program to Disseminate Tenure Tracking System from the Japan Science and Technology Agency (JST), and by the Ito Foundation.

\section{REFERENCES}

Adeyemi O.S., Adewumi I., Faniyan T.O. 2014: Silver nanoparticles influenced rat serum metabolites and tissue morphology. J. Basic Clin. Physiol. Pharmacol. 26: 355-361.

Adeyemi O.S., Faniyan T. 2014: Antioxidant status in rats orally administered silver nanoparticle. J. Taibah Uni. Med. Sci. 9: $182-186$.

Adeyemi O.S., Murata Y., Sugi T., Han Y., Kato K. 2018a: Exploring amino acid-capped nanoparticles for selective anti-parasitic action and improved host biocompatibility. J. Biomed. Nanotechnol. 14: 847-867.

Adeyemi O.S., Murata Y., Sugi T., Kato K. 2017: Inorganic nanoparticles kill Toxoplasma gondii via changes in redox status and mitochondrial membrane potential. Int. J. Nanomed. 12: 1647-1661.

Adeyemi O.S., Olajide I.O., Adeyanju A.A., Awakan O.J., Отонinoy I D.A. 2018b: Modulation of rat plasma kynurenine level by platinum nanoparticles and likely association with oxidative stress. Biointerface Res. Appl. Chem. 8: 3364-3367.

Adeyemi O.S., Sulaiman F.A. 2015: Evaluation of metal nanoparticles for drug delivery systems. J. Biomed. Res. 29: 145-149.

Adeyemi O.S., Sulaiman F.A., Akanji M.A., Oloyede H.O.B., Olatunde A., Ajayi A.G., Salman S.T., Aransiola A.R., Ekundayo M.M., Abubakar F.A., Olaoye S.A. 2016: Biochemical and morphological changes in rats exposed to platinum nanoparticles. Comp. Clin. Pathol. 25: 855-864.

Ahmad A., Syed F., Shah A., Khan Z., Tahir K., Khan A.U., YUAN Q. 2015: Silver and gold nanoparticles from Sargentodoxa cuneata: synthesis, characterization and antileishmanial activity. RSC Adv. 5: 73793-73806.

Allahverdiyev A.M., Abamor E.S., Bagirova M., Ustundag C.B., Kaya C., Kaya F., Rafailovich M. 2011: Anti-leishmanial effect of silver nanoparticles and their enhanced antiparasitic activity under ultraviolet light. Int. J. Nanomed. 6: 2705-2714.
Black M.W., Boothroyd J.C. 2000: Lytic cycle of Toxoplasma gondii. Microbiol. Mol. Biol. Rev. 64: 607-623.

Bohne W., Heesemann J., Gross U. 1994: Reduced replication of Toxoplasma gondii is necessary for induction of bradyzoite-specific antigens: a possible role for nitric oxide in triggering stage conversion. Infect. Immun. 62: 1761-1767.

Boothroyd J.C., Dubremetz J.F. 2008: Kiss and spit: the dual roles of Toxoplasma rhoptries. Nat. Rev. Microbiol. 6: 79-88.

Curtis A., Wilkinson C. 2001: Nanotechniques and approaches in biotechnology. Trends Biotechnol. 9: 97-101.

DebBage P. 2009: Targeted drugs and nanomedicine: present and future. Curr. Pharm. Des. 15: 153-172.

Jiao Z., Li M., Feng Y., Shi J., Zhang J., Shao B. 2014. Hormesis effects of silver nanoparticles at non-cytotoxic doses to human hepatoma cells. PLoS ONE. 9: e102564.

Kamau E.T., Srinivasan A.R., Brown M.J., Fair M.G., CaRAHER E.J., BOYLE J.P. 2012: A focused small-molecule screen identifies 14 compounds with distinct effects on Toxoplasma gondii. Antimicrob. Agents Chemother. 56: 5581-5590.

Leyke S., Köhler-Sokolowska W., Paulke B., Presber W. 2012: Effects of nanoparticles in cells infected by Toxoplasma gondii. E-Polymers 12: 647-663.

Maneewattanapinyo P., Banlunara W., Thammacharoen C., Ekgasit S., Kaewamatawong T. 2011: An evaluation of acute toxicity of colloidal silver nanoparticles. J. Vet. Med. Sci. 73: 1417-1423.

Maubon D., Bougdour A., Wong Y., Brenier-Pinchart M., Curt A., Hakimi M., Pelloux H. 2010: Activity of the histone deacetylase inhibitor FR235222 on Toxoplasma gondii: inhibition of stage conversion of the parasite cyst form and study of new derivative compounds. Antimicrob. Agents Chemother. 54: 4843-4850. 
Pokharkar V., Dhar S., Bhumkar D., Mali V., Bodhankar S., Prasad B.L. 2009: Acute and subacute toxicity studies of chitosan reduced gold nanoparticles: a novel carrier for therapeutic agents. J. Biomed. Nanotechnol. 5: 233-239.

Rahul S., Chandrashekhar P., Hemant B., Bipinchandra S., Mouray E., Grellier P., Satish P. 2015: In vitro antiparasitic activity of microbial pigments and their combination with phytosynthesized metal nanoparticles. Parasitol. Int. 64 353-356.

Saad A.H.A., Soliman M.I., Azzam A.M., Mostafa B. 2015 Antiparasitic activity of silver and copper oxide nanoparticles against Entamoeba histolytica and Cryptosporidium parvum cysts. J. Egypt. Soc. Parasitol. 45: 593-602.

Saini P., Saha S.K., Roy P., Chowdhury P., Sinha Babu S.P. 2016: Evidence of reactive oxygen species (ROS)-mediated apoptosis in Setaria cervi induced by green silver nanoparticles from Acacia auriculiformis at a very low dose. Exp. Parasitol. 160: 39-48.

Skariah S., McIntyre M.K., Mordue D.G. 2010: Toxoplasma gondii: determinants of tachyzoite to bradyzoite conversion. Parasitol. Res. 107: 253-260.
Sugi T., Masatani T., Murakoshi F., Kawazu S., Kato K. 2014: Microplate assay for screening Toxoplasma gondii bradyzoite differentiation with DUAL luciferase assay. Anal. Biochem. 464: 9-11.

Sulaiman F.A., Akanji M.A., Oloyede H.O.B., Sulaiman A.A., Olatunde A., Joel E.B., Adewale T.H., Adeboye H.A., Idris S.O., Quadri A.L., Oyegoke R.A., Adeyemi O.S. 2015: Oral exposure to silver/gold nanoparticles: status of rat lipid profile, serum metabolites and tissue morphology. J. Med. Sci. 15: 71-79.

Tomita T., Bzik D.J., Ma Y.F., Fox B.A., Markillie L.M., TAYlor R.C., Kim K., Weiss L.M. 2013: The Toxoplasma gondii cyst wall protein CST1 is critical for cyst wall integrity and promotes bradyzoite persistence. PLoS Pathog. 9: e1003823.

Weiss L.M., Ma Y.F., Takvorian P.M., Tanowitz H.B., WitTNER M. 1998: Bradyzoite development in Toxoplasma gondii and the hsp70 stress response. Infect. Immun. 66: 3295-3302.

Yан C.S., Simate G.S. 2015: Nanoparticles as potential new generation broad spectrum antimicrobial agents. Daru. 23: 43

Cite this article as: Adeyemi O.S., Murata Y., Sugi T., Han Y., Kato K. 2019: Nanoparticles show potential to retard bradyzoites in vitro formation of Toxoplasma gondii. Folia Parasitol. 66: 001. 